\title{
A Comparative Study of Benefits and Drawbacks of Dispensing the Exact Number of Pills
}

\author{
Srirupa Biswas* and Arumugam Balasubramaniam \\ School of Pharmacy, ITM University, India
}

Received: February 16, 2018; Published: February 26, 2018

*Corresponding author: Srirupa Biswas, School of Pharmacy, ITM University, Turari campus, AH-43 Bypass, Jhansi Rd, Gwalior, Madhya Pradesh, India 475001, Email: srirupabiswas.pharma@itmuniversity.ac.in

\begin{abstract}
Background: From August 2016 to August 2017, an experiment in Gwalior local private and government pharmacies replaced traditional pre-packed boxes by per-unit dispensing of pills in the exact numbers prescribed, for 14 antibiotics.

Methods: A cluster randomized control trial was carried out in 10 pharmacies. 5 pharmacies counted out the medication by units (experimental group), the other 5 providing the treatment in the existing Pharmaceutical company boxes (control group). Data on patients under the two arms were compared to assess the epidemiological, economic and health effects of this change in drug dispensing. In particular, Adherence was measured indirectly by comparing the number of pills left at the end of the prescribed treatment.
\end{abstract}

Results: Out of the 1185 patients included during 3 sessions of 4 consecutive weeks each, 907 patients experimented the personalized delivery and 278 were assigned to the control group, consistent with a 1/3 randomization-rate at the pharmacy level. Patient consent was taken before assigning in each group. The initial packaging of the drugs did not match with the prescription in $60 \%$ of cases and per-unit dispensing reduced by $10 \%$ the number of pills supplied. $13.1 \%$ of patients declared that they threw away pills residuals instead of recycling Finally, perunit dispensing appeared to improve adherence to antibiotic treatment (marginal effect 0.21 , IC 95, 0.14-0.28).

Conclusions: Supplying antibiotics per unit is not only beneficial in terms of a reduced number of pills to reimburse or for the environment (less pills wasted), but also has a positive and unexpected impact on adherence to treatment, and thus on both individual and public health and has an epidemiological impact.

Keywords: Epidemiological Impact; Drug Dispensing; Economic; Pre-Packed Boxes; Per-Unit Dispensing

\section{Introduction}

\section{Background}

Different pharmacies in Gwalior vary in their methods of supplying drugs, some dispensing packaged drugs, others dispensing the exact number of tablets required. The healthcare system, and in particular the method of supplying antibiotics, has been shown to impact the risk of misuse of drugs, for example through self medication [1]. Yet, to the best of our knowledge, a proper comparison between these two dispensing systems has not been undertaken. The recent and sustained development of bacterial resistance [2]. has prompted many countries to combat this major public health problem. India \& France has high indicators of antibiotic resistance in numerous domains (e.g.: staphylococcus pneumonia, with tested susceptibility to penicillin or macro ides) [3]. Which can be explained by the very high prescription rates for antibiotics and plausible over use or misuse of these drugs [4]? After a decrease in the early 2000s, France recently experienced an increase in antibiotic consumption [5].
While the pharmaceutical industry usually argues that packaging ensures traceability and provides better patient information and safety, this dispensing mode has the disadvantage of not always matching the prescription. This can lead to losses for the government national healthcare system, misuse of antibioticsfor example, self-medication or overlong treatment, as well as surplus pills being released into the environment causing toxicity. On the level of society as a whole, dispensing the exact number of pills required by the medical prescription may be one way to avoid these potential effects. Through a controlled intervention among private and government local pharmacies in Gwalior over one year, we assessed the Feasibility and the real impact of a change in the method of dispensing antibiotics in Gwalior community Pharmacies for 14 antibiotics (Appendix A). This article reports the first results of this Experimental intervention study. Assessed outcomes were: number of pills saved, potential gains for the Environment and adherence to treatment. 


\section{Methods}

\section{Study Design}

Throughout 2016-17, a clustered randomized controlled trial was implemented in 10 pharmacies selected randomly from the 27 that volunteered to participate, as invited through a telephonic call. Pharmacies were allocated, based on a random number picked. 5 pharmacies (control group) continued providing antibiotics in the usual standard packaging from pharmaceutical companies, while other 5 pharmacies (intervention group) dispensed the exact number of pills to patients with a prescription for the eligible antibiotics.

\section{Sample Size Computation}

At the time of the protocol deposit, there was still some uncertainty regarding the number of Participating pharmacies, and thus on the number of clusters. For this reason, a sample size recalculation was not possible. Fernandez et al. [6]. Studied adherence behavior in Portugal with Roughly 350 outpatients recruited in community pharmacies. In order to be on the safe side, we estimated that 700 subjects were needed to estimate a significant difference in the main outcome variables that we intend to deal with. In the course of the experiment, we were able to increase the sample size up to 1185 respondents.

\section{Data Collection}

This design was coupled with an observational study of pharmacists, to assess the percentage of cases where the repacked boxes were not matching the prescribed number of pills as well as, in each case, the precise number of pills supposedly wasted if pre-packed boxes were used. The pharmacists recruited in this experiment were given study-related training: understanding of the protocol, rules for data collection, and modalities of patient recruitment. The protocol required pharmacists to recruit patients in a systematic way. First, they asked all patients with an antibiotic prescription whether they agreed with unit dispensing. Then, whatever the response, they invited patients to participate in a telephone survey.

Out of the 1,731 recruited by pharmacy staff, 1,238 patients actually agreed to be interviewed by phone by independent interviewers two t o three days (on average) after completion of their treatment (attrition rates are equivalent in the two arms -T-test of differences with p-value $=0.34$ ). In addition to questions about acceptance of the dispensing mode, the research team collected data on habits of disposal of drugs, intended self-medication, quality of the information provided by the pharmacy staff for the current antibiotic treatment and adherence to this treatment.

\section{Main Outcome Variables}

Outcome variables relate to economic concerns: the number of pills saved in the per-unit delivery mode; but also to social and public health benefits: acceptance from patient; quality of information received at the pharmacy; adherence behavior. To this end, respondents were questioned retrospectively about the whole course of their antibiotic treatment, from pharmacy dispensing, to end of treatment (generally at home). First, the number of pills they received, together with the medical prescription, was recorded by the pharmacist. Second, the number of pills left after completion of treatment was collected through the patient's survey. Using information on the number of pills provided by the pharmacies, we are able to compute the number of pills left in the two arms of the study, for a comparative purpose. For patients in the control group, the information was corrected in order to exclude the useless extra pills received by those patients; e.g., for one patient receiving a box of 30 pills for a treatment of 3 pills per day for 8 days (a real need of 24).

We systematically correct his declaration of pills-left after completion by 6 (30-24) which was an extra amount NOT relevant for the comparison. We then inferred the patient's "adherence" by comparing the expected and the actual number of pills left after completion of treatment (a similar counting method was used, for example, by [7]. to measure adherence to antibiotic treatments in outpatients' daily life). Finally, a standardized and validated scale [8]. Of adherence, named the Moresby scale, was introduced in the questionnaire. From all this, we defined three distinct indicators of patients' adherence: no pills left (strict adherence criterion), less than four pills left (one-day tolerance criterion).

\section{Data Analysis}

The randomization of pharmacies ensured not only the comparability of patients' individual characteristics in the two arms, but also unbiased assessment of the impact of the change in antibiotic dispensing mode. We checked the quality of the randomization by T-test. The outcomes described above number of pills supplied, quality of information received at the pharmacy) are compared, for the two arms of the intervention by descriptive statistics and T-test. The adherence outcome is compared through a probit regression with robust standard errors clustered at the pharmacy level and sample selection equation (Heckman). This identification strategy allows ensuring that there is no sampleeffect

\section{Ethics and Trial Registration}

The study is not a classical clinical trial, i.e. competition between different types of drugs-with possible severe impact on the health of the patient, but a simple modification in the delivery mode of the same drug. In that case, the Indian law does not constrain to register the study as a clinical trial. All patients receiving the exact number of pills required signed an informed consent certifying they agreed on the dispensing mode of their treatment. All patients interviewed signed an additional consent to indicate that they agreed on the telephone survey participation.

\section{Results}

\section{Per-unit Dispensing Acceptance}

The patient acceptance rate for per-unit dispensing was $80.6 \%$ (computed over the whole sample of eligible patients, thus eliminating any possibility of bias from agreeing to the phone survey). Of eligible patients, $39.3 \%$ agreed to take part in the 
phone survey. Individuals who refused the per-unit dispensing were excluded from the analysis living us 1,185 out of the 1,238 interviewed participants (Appendix B).

\section{Economic Consequences}

The initial drug packaging had to be modified in $60.0 \%$ of cases of per-unit dispensing, meaning that the packaging available in the community pharmacy did not match the prescription (or the reverse: the prescription did not match the pre-packed box). Dispensing the exact number of drugs reduced by $9.9 \%$ the number of pills supplied. Information provided by the pharmacist Table 1: Patient and community pharmacy characteristics.
Respondents were asked whether the pharmacist who attended them provided a series of information regarding their treatment (treatment length, number of doses per day, recycling mode, risk of no adherence, among others). No difference in information given to patients regarding appropriate use of their medication was found between the two groups Self-medication and recycling issue In the phone survey, $17.6 \%$ of respondents declared their intention to keep the antibiotics left after completion of treatment. $10.7 \%$ of respondents also admitted that they could use antibiotics without the consent of a physician (Table 1).

\begin{tabular}{|c|c|c|c|}
\hline & Control group & Treated group & p-value \\
\hline Variables & \% (X/N) [SE] & \%, (X/N) [SE] & \multicolumn{2}{|c|}{0.56} \\
\hline \multicolumn{2}{|c|}{ Randomization at patient level-excluding individuals who refused per-unit dispensing } \\
\hline Female gender & $60.8(169 / 278)$ & $62.7(569 / 907)$ & 0.22 \\
\hline Age of adult patients (in years) $a$ & $52.8(246)[17.0]$ & $54.3(862)[17.0]$ & 0.47 \\
\hline Low socio-economic status & $52.9(129 / 244)$ & $50.2(392 / 781)$ & 0.91 \\
\hline Has children & $81.8(224 / 274)$ & $81.4(728 / 894)$ & 0.14 \\
\hline Married & $59.3(162 / 273)$ & $54.3(483 / 890)$ & 0.25 \\
\hline Employed & $53.1(145 / 273)$ & $49.1(434 / 884)$ & 0.39 \\
\hline
\end{tabular}

The reduction in residuals induced by the per-unit delivery could lead to a decrease in self-medication of $1.9 \%(10.7 \% \mathrm{x}$ $17.6 \%)$. Last, $13.1 \%$ reported that they generally throw away their pills. Adherence Per-unit dispensing also appeared to improve adherence to the antibiotic treatment. A patient was considered as "adherent" if he had no pills left at all (intervention group), and Table 2: Descriptive statistics of adherence to treatment. after excluding extra pills received due to a prescription / packaging mismatch (control group). The pill count information is available for 984 respondents. $65.6 \%$ and $91.4 \%$ of patients were adherent (strict adherence criterion) in the control and the intervention groups respectively (Appendix C) (Table 2).

\begin{tabular}{|c|c|c|c|}
\hline & Control group & Treated group & p-value \\
\hline Variables & $\%(\mathbf{X} / \mathbf{N})$ & \%, (X/N) & 0.00 \\
\hline Strict adherence criterion & $65.6(84 / 128)$ & $91.4(782 / 856)$ & 0.00 \\
\hline One day tolerance criterion & $71.1(91 / 128)$ & $92.3(790 / 856)$ & 0.00 \\
\hline Mixed adherence criterion & $57.5(73 / 127)$ & $77.8(660 / 848)$ & \\
\hline
\end{tabular}

\section{Descriptive Statistics of Adherence to Treatment}

From (Table 3), presenting multivariate regressions, we see that the probability of being adherent is always greater in the intervention group. Marginal effects ranged from 0.23 [0.15; 0.31] to 0.21 [0.14; 0.28$]$ depending on the measures (with 95\% interval confidence in bracket). This first value of 0.23 Means that patients under the per-unit delivery system are estimated 23 percentage- points more likely to be adherent to their antibiotic treatment. Robustness tests involved

a) Taking a looser indicator of patient adherence: having less than a day's medication left and

b) Combining the count with a standardized scale of adherence [8] (Table 3).

Table 3: Impact on adherence of dispensing exact quantities required.

\begin{tabular}{|c|c|c|c|}
\hline & $\begin{array}{c}\text { Strict adherence criterion (no } \\
\text { pills left) }\end{array}$ & $\begin{array}{c}\text { One day tolerance criterion (less } \\
\text { than four pills left) }\end{array}$ & $\begin{array}{c}\text { Mixed adherence criterion } \\
\text { (counting method combined with } \\
\text { the Morisky scale) }\end{array}$ \\
\hline Treated & $0.23^{*}$ & $0.20^{*}$ & $0.21^{*}$ \\
\hline Pharmacies & {$[0.15 ; 0.31]$} & {$[0.12 ; 0.29]$} & {$[0.14 ; 0.28]$} \\
\hline $\begin{array}{c}\text { Excluding individuals who refused } \\
\text { per-unit dispensing }\end{array}$ & Yes & Yes & Yes \\
\hline
\end{tabular}




\begin{tabular}{|c|c|c|c|}
\hline Observations & 1185 & 1185 & 1185 \\
\hline Censored observations & 984 & 984 & 975 \\
\hline
\end{tabular}

Impact on adherence of dispensing exact quantities required. Control variables are age and type of treatments; they were

introduced after a stepwise procedure which determines their significance at the critical p-value of 0.10 (Figure 1).

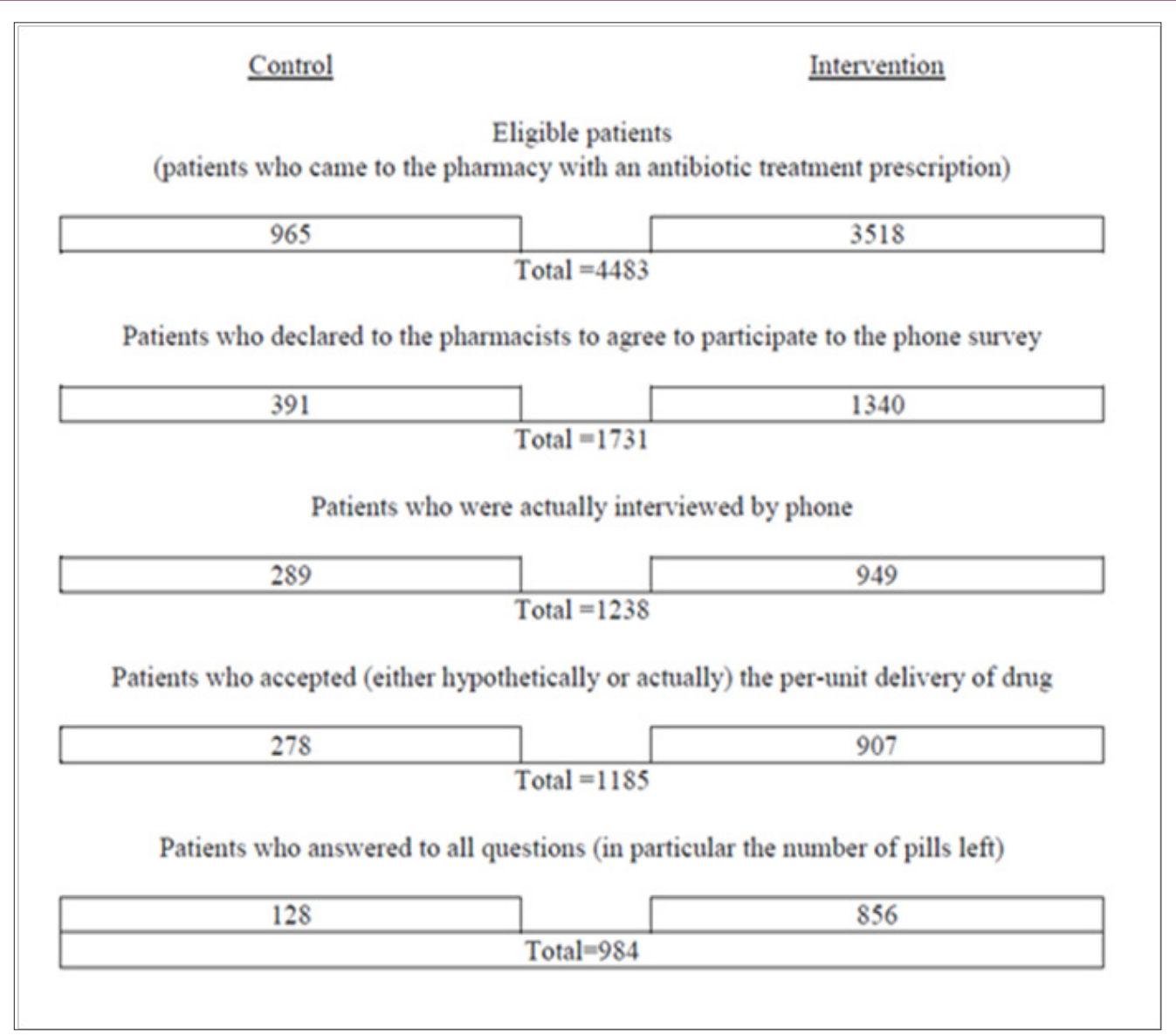

Figure 1.

\section{Discussion}

\section{Major Findings}

This study investigates the impact of per-unit dispensing of 14 antibiotics on three outcomes likely to generate antibiotic resistance: self-medication, surplus pills being released into the environment and compliance with treatment [3-4,9]. We find allround beneficial effects. First, lower quantities of antibiotics are supplied under the per-unit dispensing system. This confirms that, despite the claims of the pharmaceutical industry or of retail pharmacists, there is a degree of mismatch between prescriptions and packaging (this may be either due to the drug dispensing system or the physician, who may prescribe the wrong quantity or wrong treatment period). This mismatch suggests a potential source of savings for the Indian national healthcare system that reimburses prescribed medicine (as, for each pill delivered in excess in the traditional delivery mode, there is waste of financial resources-however see the dedicated paragraph below for possible counterbalancing limitations and costs).
Second, the lower likelihood of having pills left at the end of treatment (often not recycled) may also reduce self-medication and bacterial resistance, consistent with findings by Grigoryan and coauthors [1]. Supplying antibiotics per unit might thus have long-term positive effects on the environment, although, because restricted to the (only) indirect evidence of probable release of pills in the nature, the design of the study does not permit us to assess these delayed effects in a precise quantitative manner (ecological indicators of bacterial resistance). Third, dispensing the exact number of pills needed appears to increase adherence to the treatment. Although the beneficial economic and environmental effects of this type of drug supply were anticipated, the improvement of adherence was not. While pharmaceutical companies defend their pre-packed drug packaging, this does not always seem to be an efficient way of dispensing antibiotics, in economic, environmental and patient's health terms. Note that the benefits from adherence can also be linked with parallel outcomes related to the population's health: non-adherence to antibiotic treatment leads to therapeutic failure, re-infection and bacterial resistance $[3,4,9]$. 


\section{Adherence Measurement Limitations}

We acknowledge that our measurement of adherence relies on patients' declarations, which may be a limitation. While memory bias is limited due to the short lapse of time between completion of treatment and the interview, a social desirability bias may arise when patients declare how many pills they have left (more likely in the intervention group). However, only indirect measures are possible with outpatients and with such acute diseases. It might be possible, by observing data on health expenditure reimbursements, to identify the dispensing of an equivalent drug a few weeks after completion of the initial treatment (a second similar prescription could indicate poor compliance). Such data have not been obtained yet. Nevertheless, robustness tests combining two validated tools encourage some confidence in our results. Finally, the random design of the study should ensure that any measurement errors are equally distributed in the two experimental arms (for adherence behaviours and the other outcomes considered).

\section{Other limitations}

Other weaknesses of the study were the following: participants were not asked about the diagnosis associated with the antibiotic prescription (this would imply a cross-validation by the physician which was difficult to set in place); the participation to the phone survey was disappointing (but equal in the two arms); delayed effects on the environment (bacterial resistance) are not assessable in this small scale study; and last, we had to renounce to make a standard cost-benefit evaluation study, because equilibrium prices, fees and costs appeared too complex to estimate in this preliminary phase of the policy.

\section{Beyond the Study Itself}

The cost of a change in dispensing, from the current pre-packing in boxes to a per-unit system, lies in an increased workload for pharmacists, not evaluated in this study (since no adjustment for per-unit dispensing had been made to the drug provision chain, due to the small scale of the intervention). We recognize that the absence of a precise accountability of these labour costs is an obstacle for the assessment of the complete "financial" consequences of the policy. But overall its benefecial for any society to reduce wastage of important and costly drugs and garbage.

\section{Acknowledgment}

We would like to thank the ITM University, Gwalior, local private and government pharmacies and participating patients and pharmacists of Gwalior.

\section{References}

1. Grigoryan L, Burgerhof JG, Degener JE, Deschepper R, Stalsby Lundborg C, et al. (2008) Determinants of self-medication with antibiotics in Europe: the impact of beliefs, country wealth and healthcare system. Journal of Antimicrobial Chemotherapy 61(5): 1172-1179.

2. Mayor S (2005) Antibiotic resistance is highest in south and east Europe. BMJ 330(7488): 383.

3. (2015) European Centre for Disease Prevention and Control. Antimicrobial resistance surveillance in Europe 2014. Annual Report of the European Antimicrobial Resistance Surveillance Network (EARSNet). ECDC, Stockholm, Sweden.

4. Goosens H, Ferech M, Stichele RV, Elseviers M (2005) Outpatient antibiotic use in Europe and association with resistance: a cross-national database study. Lancet 365(9459): 579-587.

5. (2014) Agence Nationale de Sécurité du Médicament et des produits de santé (ANSM). L'évolution des consommations d'antibiotiques en France entre 2000 et 2013 . ANSM.

6. Fernandes M, Leite A, Basto M, Nobre MA, Vieira N, et al. (2014) Non-adherence to antibiotic therapy in patients visiting community pharmacies. International Journal Clin. Pharm 36(1): 86-91.

7. Suffoletto B, Calabria J, Ross A, Callaway C, Yealy DM (2012) A Mobile Phone Text Message Program to Measure Oral Antibiotic Use and Provide Feedback on Adherence to Patients Discharged From the Emergency Department. Academic Emergency Medicine 19(8): 949-958.

8. Morisky DE, Lawrence WG, Levine DM (1986) Concurrent and predictive validity of a self-reported measure of medication adherence. Medical Care 24(1): 56-74.

9. Wahl LM, Nowak MA (2000) Adherence and drug resistance: predictions for therapy outcome. Proceedings of the Royal Society of London B: Biological Sciences 267(1445): 835-843.
This work is licensed under Creative Commons Attribution 4.0 License

Submission Link: http://biomedres.us/submit-manuscript.php

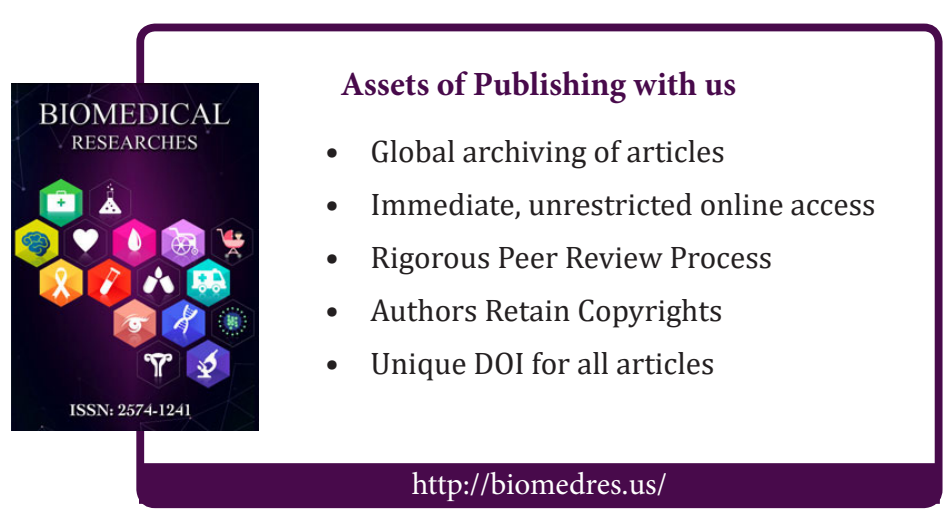

